\title{
Developmental aspects of schizophrenia and related disorders: possible implications for treatment strategies
}

\author{
Eric J. Davies
}

Abstract Schizophrenia and other schizophrenia-spectrum disorders are neurodevelopmental disorders which may share genetic susceptibility factors and represent differential expressions of an underlying vulnerability. Schizophrenia may have its onset in childhood and can be reliably diagnosed. However, developmental factors modulate disease expression in children. Although the prevalence of schizophrenia in childhood is low, children who develop schizophrenia in adult life may show subtle and non-specific developmental abnormalities, consistent with the neurodevelopmental hypothesis. Studies of the schizophrenia prodrome also demonstrate that abnormalities may be apparent years before the onset of positive symptoms. Such evidence raises the possibility of using preventive approaches in the treatment of schizophrenia. Further advances in our knowledge of the aetiopathology of schizophrenia (and the identification of endophenotypes within the group of schizophrenia and related disorders) may further improve our ability to predict disease development, making implementation of preventive interventions more achievable.

Schizophrenia and schizophrenia-spectrum disorders are neurodevelopmental in origin and are likely to represent differential expression of an underlying vulnerability. Children who develop schizophrenia in adult life may show non-specific developmental abnormalities. Moreover, studies of the schizophrenia prodrome show that abnormalities are observed years before the onset of positive symptoms. This article describes developmental aspects of schizophrenia and related disorders and the implications of these findings for assessment and early intervention.

\section{Clinical features}

\section{Schizophrenia}

Schizophrenia is a chronic psychiatric disorder, with a lifetime risk of about $1 \%$. Its clinical features can be conceptualised in a number of ways. One of these is their broad division into positive and negative symptom clusters. Alternatively, the characteristic symptoms of schizophrenia can be divided into three syndromes or dimensions of illness (Liddle, 1987) as in Box 1: reality distortion (delusions and hallucinations); psychomotor poverty (negative symptoms); and disorganisation (thought disorder). In addition to the clinical symptoms, a range of neurocognitive abnormalities may be present and may be associated with functional outcome. These include deficits in attention, verbal memory, working memory and executive function. These deficits may be antecedents, rather than consequences, of the illness.

Most populations show similar prevalence rates of schizophrenia of between 1.4 and 4.6 per 1000 . Incidence rates for the disorder are dependent on the diagnostic criteria used to ascertain caseness (Jablensky et al, 1992).

\section{Schizotypy}

Schizotypy (or schizotypal personality disorder), like schizophrenia, is syndromal in nature. The three main components of the syndrome mirror the abnormalities seen in schizophrenia. DSM-IV criteria specify the need for a 'pervasive pattern of social deficits', 'cognitive or perceptual distortions' and 'eccentricities' of behaviour (American Psychiatric Association, 1994). These abnormalities must not occur exclusively in the context of schizophrenia, affective disorder with psychotic features, other psychotic disorder or a pervasive developmental disorder.

The prevalence of schizotypy is higher in clinical than in non-clinical samples, and the prevalence within the families of people with schizophrenia is higher than the population prevalence, at about $10 \%$ (Kendler \& Gardner, 1997).

Eric Davies is Consultant Child and Adolescent Psychiatrist working with the 5 Boroughs Partnership NHS Trust (The Elms, 50 Cowley Hill Lane, St Helens, Merseyside WA10 2AW, UK. Email: eric.davies2@5bp.nhs.uk). He trained at the University of Manchester, where he also completed a $\mathrm{PhD}$ on factors related to genetic susceptibility in systemic lupus erythematosus. He has a particular interest in the genetics of schizophrenia and is an Honorary Research Fellow in the Division of Psychiatry at the University of Manchester. 


\section{Box 1 Clinical features of schizophrenia and schizotypy}

Schizophrenia

- Reality distortion (delusions and hallucinations)

- Psychomotor poverty (negative symptoms)

- Disorganisation (thought disorder)

Schizotypy

- May have transient psychotic experiences

- Negative features (social withdrawal, affective blunting)

- Cognitive disorganisation

- Have never met diagnostic criteria for schizophrenia

Schizotypy includes some of the features of schizophrenia and schizoaffective disorder (Box 1) and is often apparent by early adulthood. However, schizotypal personality traits may be apparent in childhood or adolescence. A major factor distinguishing schizotypy from schizophrenia and other psychoses is the transient nature of psychotic experiences.

People with schizotypy often demonstrate poor social interactions. In addition to this and their attenuated psychosis-like experiences, they often show deficits in cognitive function, which are similar to those seen in schizophrenia but less severe. Schizotypy and schizophrenia tend to co-occur in families, suggesting that there may be shared susceptibility factors. However, schizotypy does not occur only as a precursor to schizophrenia. Rather, it appears to be an alternative expression of an underlying vulnerability which may or may not herald the onset of schizophrenia.

\section{The schizophrenia spectrum}

Diagnostic criteria for schizophrenia are necessarily somewhat arbitrary owing to limitations in our knowledge of the aetiopathology of the disorder. This leads to dilemmas regarding the definition of boundaries and the recognition that many of the clinical features of schizophrenia are dimensionally distributed.

It is recognised that schizophrenia and schizotypy share some genetic susceptibility factors. In addition, twin studies suggest that a predisposition to disease is transmitted but is not necessarily expressed as schizophrenia. Family studies indicate that a range of clinical conditions are more frequent in the relatives of people with schizophrenia (Lichtermann et al, 2000). These include schizoaffective disorder, atypical and schizophreniform psychoses, affective psychoses with mood-incongruent delusions as well as schizotypal personality disorder (Box 2). Thus it is

\section{Box 2 Features of the schizophrenia spectrum}

- Several disorders cosegregate with schizophrenia in families:

- schizoaffective disorder

- schizotypy

- atypical and schizophreniform psychoses

- affective psychosis with moodincongruent delusions

- Shared genetic susceptibility factors between schizophrenia and schizotypy

- Neuropsychiatric defects in clinically unaffected first-degree relatives of people with schizophrenia

- Concept of schizotaxia as inherited susceptibility

- More recent concept of cognitive dysmetria

possible that shared susceptibility factors may result in the expression of a variety of clinical phenotypes. These disorders can be considered as parts of the schizophrenia spectrum, a constellation of related but clinically diverse conditions.

\section{Genetic aspects of schizophrenia and schizotypy}

It is well established that schizophrenia has a sizeable genetic component and that schizotypy may share common genetic vulnerability with schizophrenia. Meehl (1989) postulated the concept of schizotaxia, an underlying genetic vulnerability to both schizophrenia and schizotypy. Schizotaxia is apparent in $20-50 \%$ of first-degree relatives of people with schizophrenia. It is thus clear that the underlying genetic vulnerability does not necessarily lead to either schizophrenia or schizotypy. Studies have shown that clinical symptoms in first-degree relatives of people with schizophrenia include negative symptoms which are similar in nature to (but less severe than) those seen in schizophrenia (e.g. Kendler et al, 1995). Positive symptoms tend to be less evident than in schizophrenia or schizotypy, but neuropsychological impairments are also seen. Schizotypal features are more common in first-degree relatives of people with schizophrenia and mirror the symptom dimensions of schizophrenia. Updating the concept of schizotaxia, Andreasen (1999) suggested a view of schizophrenia as involving abnormal connections in the cortico-cerebellar-thalamic-cortical circuit (normally used to coordinate both motor and mental activity). These lead to misconnections in many aspects of mental activity, or 'cognitive dysmetria'. It has recently been reported that the 
anatomical changes underlying cognitive dysmetria may also underlie the early developmental abnormalities seen in schizophrenia (Ridler et al, 2006).

Genetic studies of schizophrenia have suggested several chromosomal regions that appear to be linked with the disorder (Berry et al, 2003). Findings from investigations of candidate genes have often not been consistently replicated. However, there have been recent advances, with the identification of several putative susceptibility genes that may be involved in synaptic function and organisation (Harrison \& Weinberger, 2005). These findings provide support for the neurodevelopmental hypothesis of schizophrenia, as well as providing a possible link between genetic susceptibility factors and the observed neuroanatomical abnormalities. Interest has also focused on the possible role of epigenetic factors in schizophrenia (i.e. there may be factors other than specific gene sequence that can affect the way a disorder is inherited) and on the identification of particular endophenotypes (Gottesman \& Gould, 2003) under genetic control (Berry et al, 2003).

\section{Developmental aspects}

\section{Schizophrenia}

Epidemiological studies demonstrate that the onset of schizophrenia is age-related (Hafner et al, 1993; Kirkbride et al, 2006). There is an increase in onset after puberty, continuing throughout adolescence and peaking in the twenties. Onset then decreases from the thirties and tends to tail off in the fifties. In the $Æ$ ESOP study (Kirkbride et al, 2006), more than $76 \%$ of men and $63 \%$ of women with psychosis had made first contact with mental health services before the age of 35. The pattern of onset may reflect the neurodevelopmental processes important in the development of schizophrenia, and highlights the somewhat arbitrary divisions in the organisation of mental health services (into child and adolescent mental health services and adult psychiatric services) when considering schizophrenia.

However, schizophrenia can occur in children (Rapoport et al, 1997; Hollis, 2000) and developmental factors modulate the expression of the disease and the ability to describe symptomatology (Box 3). Younger children without any psychiatric disorder tend to score more highly on thought disorder indices than older children (Asarnow et al, 2004). It is therefore important to consider developmental level when interpreting results, and longitudinal data are important in confirming diagnoses. Despite this, a diagnosis of schizophrenia in childhood does have predictive validity (Hollis, 2000). In children it is essential to rule out possible organic causes for the presentation of schizophrenia-like illnesses, including neurological and metabolic disorders.
Childhood-onset schizophrenia can present with a gradual and insidious onset, leading to the question of when the diagnosis of schizophrenia should be made. Moreover, a high proportion of children with schizophrenia meet diagnostic criteria for another psychiatric diagnosis, highlighting the important issue of comorbidity (Asarnow et al, 2004).

The consensus is that childhood- and adult-onset schizophrenia are the same disease entity. However, some studies suggest possible differences according to age at onset; for example, patients with earlyonset schizophrenia showed reduced hemispheric differentiation compared with patients with lateonset schizophrenia (Asarnow et al, 2004).

Studies of the outcome of schizophrenia in adults have shown variable results, a situation mirrored in studies of childhood schizophrenia (Bellgrove et al, 2004). The outcome of childhood schizophrenia is generally poorer than that of adult-onset schizophrenia. Problems are observed with social impairments and ongoing psychotic symptoms and need for psychiatric services. Hollis (2000) found that early-onset schizophrenia had a poorer prognosis than non-schizophrenic psychoses. This included greater negative and positive symptoms, less time in remission, lower educational attainment, less time in work, more time in residential care and greater social isolation. Several studies report poorer outcomes in children with poor adjustment prior to the onset of schizophrenia. Negative symptoms have been associated with poorer outcomes in some studies of childhood schizophrenia (Asarnow et al, 2004).

Despite some general differences from adult-onset schizophrenia, schizophrenia does occur in children and can be reliably diagnosed using adult criteria. However, this only accounts for a small proportion of the total number of incident cases of schizophrenia. The question then arises as to whether there are any features which may help to identify those children who will develop schizophrenia in adult life.

\section{Box 3 Developmental expression of schizo- phrenia and schizotypy}

- Most people with schizophrenia have onset in late adolescence or adulthood, but onset in childhood does occur

- In children, clinical phenotype is modulated by developmental factors

- Outcome of childhood-onset schizophrenia is usually worse than that of adult-onset

- Schizotypal features can become apparent in childhood

- People who develop schizophrenia may exhibit a range of premorbid subtle developmental abnormalities 


\section{Schizotypy}

Schizotypal personality traits can become apparent during childhood or adolescence. Adolescents scoring highly for schizotypy may perform poorly on neurocognitive measures, have more soft neurological signs and higher teacher report ratings of behavioural problems compared with their peers, which is consistent with studies of schizotypy in adults. Asarnow et al (1994) published an outcome study of 12 young people presenting with schizotypal disorders. A high proportion met criteria for an ongoing schizophrenia-spectrum disorder. Schizotypy was the most common disorder observed, although one of the sample developed schizophrenia during the follow-up period and two developed schizoaffective disorder.

\section{Schizophrenia prodrome}

People who develop schizophrenia show a range of problems before the onset of frank psychosis. Although studies have often concentrated on positive symptoms, negative symptoms do occur and may relate to later functional disability. Constituents of the prodrome include cognitive deficits which may precede other symptoms by years, affective disturbances, social isolation, deficits in social functioning and school failure. Studies support the hypothesis that the underlying vulnerability to disorders in the schizophrenia spectrum begins to manifest with negative and non-specific symptoms (Hafner et al, 1993; Cornblatt et al, 2003; Singh et $a l, 2005)$. Individuals may then progress through attenuated positive symptoms to frank psychosis.

This is in keeping with the pattern of onset of schizophrenia in children and adolescents. The question arises as to whether (and how) such individuals should be treated, particularly since most people with such features will not develop schizophrenia. Given that the earliest features apparent are not positive symptoms, interventions aimed at improving cognitive function or mood disturbance/social isolation may be most appropriate.

People who develop a psychotic illness may show a variety of structural brain changes (Pantelis et al, 2003). A number of mechanisms have been suggested to explain such progressive changes during adolescence. Genetic factors could act via changes in the expression of genes important in neurodevelopment, whereas hormonal changes occurring in adolescence could affect processes such as myelination and synaptic pruning. Psychosocial factors might also be involved in neurodevelopmental changes. Further longitudinal studies of people with the schizophrenia prodrome might lead to greater understanding of neurodevelopmental factors involved and aid treatment. However, the features of the prodrome are non-specific and the prodrome is a retrospective concept (identified after the onset of illness). Given this, early intervention studies have often focused on identifying those people who are at highest risk of transition to psychosis.

\section{Neurodevelopmental aspects and developmental precursors of schizophrenia}

There is strong evidence that schizophrenia is a neurodevelopmental disorder. Changes in brain structure have been reported in numerous studies of schizophrenia (Keshavan et al, 2005). One focus of epidemiological studies has been the premorbid state and the definition of factors present during childhood or adolescence which might be associated with later onset of disease. This contrasts with the prodrome, which can be seen as comprising the early manifestations of the onset of disease.

\section{Follow-back studies}

It has long been recognised that people who develop schizophrenia may present as 'odd' in childhood (Bleuler, 1911). Follow-back studies, although susceptible to recall bias, do suggest a pattern of early abnormal development. Analysis of school reports of people with schizophrenia showed differences in childhood social behaviour (Watt, 1978) in a reconstructed cohort. Weinberger et al (1980) reported that patients with schizophrenia and computed tomographic changes suggestive of brain atrophy had worse premorbid adjustment in childhood than those without such changes. Later studies by the same group resulted in the development of instruments assessing premorbid personality (Cannon-Spoor $e t$ $a l, 1982)$. Review of home videos suggested a higher frequency of abnormal neuromotor development in children who went on to develop schizophrenia compared with their siblings without schizophrenia (Walker, 1994).

\section{High-risk populations}

Other methods of examining the premorbid state include the use of high-risk populations defined according to family history, attenuated or brief psychotic symptoms and the use of birth cohorts.

Studies of the children of mothers with schizophrenia suggest that early development is frequently abnormal. Fish et al (1992) showed that 6 of 12 high-risk infants showed schizotypal or paranoid personality traits and 1 developed schizophrenia. They also described a pattern of abnormalities seen in the first 2 years of life as 'pandysmaturation'. 
Studies have shown some evidence of persistently abnormal motor development in high-risk infants (Erlenmeyer-Kimling et al, 2000) and of a high frequency of soft neurological signs. Davalos et al (2004) demonstrated significant deficits in verbal skills, working memory and inhibition in children at high genetic risk of schizophrenia. Johnstone et al (2005) reported findings from the Edinburgh High Risk Study in which high-risk individuals who developed schizophrenia differed from those who did not on measures of social anxiety, withdrawal and other schizotypal features.

\section{Cohort studies}

Cohort studies are less susceptible to recall bias than follow-back studies. Adults with schizophrenia show a greater frequency of problematic social interactions in childhood and adolescence than adults without schizophrenia (Done et al, 1994; Crow et al, 1995; Malmberg et al, 1998). Studies suggest that children who later develop schizophrenia exhibit an increased frequency of speech and language problems, delayed motor milestones, poor motor skills and coordination, lower premorbid IQ, educational difficulties, social anxiety and preference for solitary play (Jones et al, 1994; Malmberg et al, 1998). Cannon et al (2000) found that patients with schizophrenia and their unaffected siblings demonstrated greater cognitive dysfunction during childhood than controls. These deficits in motor and cognitive domains, which are apparent early in life, are consistent with the neurodevelopmental model of schizophrenia.

\section{Neurocognitive and neurophysiological markers}

Studies of neurocognitive and neurophysiological markers as possible risk factors for schizophrenia have reported several associations. The deficits are also apparent in some asymptomatic biological relatives of people with schizophrenia (but rare in unrelated controls), which is consistent with Andreasen's concept of cognitive dysmetria (Andreasen, 1999). They include deficits in sustained attention, eventrelated brain potentials and saccadic eye movement control. If these reports are replicated in studies with greater power, they could have significant implications for risk prediction.

Monozygotic twins who develop schizophrenia have been reported to have an increased frequency of minor physical abnormalities compared with their non-affected twins, suggesting that abnormalities are present during fetal development (Bracha et al, 1991). Abnormal development may be genetically determined in some instances, but in others will be due to chance events. However, abnormal development does not in itself guarantee the later development of schizophrenia. Random environmental insults (such as birth trauma, infection or malnutrition) may subsequently act to increase the risk of later development of schizophrenia. Epigenetic mechanisms such as DNA methylation might also play a role.

Such a model, with neurodevelopmental abnormalities providing a substrate on which schizophrenia can occur, would help to explain the concordance rates observed in monozygotic twins, the clinical heterogeneity of schizophrenia and the different phenotypic expression of underlying genetic vulnerability within families. Furthermore, longitudinal imaging studies suggest that anatomical changes occur in the early stages of schizophrenia, which indicates that later neurodevelopmental influences may also be involved in the genesis of the disorder.

\section{Neuroplasticity}

There has been recent interest in the possible contribution of neuroplasticity (Frost et al, 2004). Neuroplasticity phenomena result in change in neural structure and function; they are involved in normal brain development and also operate in disease. This approach may help to explain the heterogeneity of schizophrenia (in all domains, including clinical phenotype, treatment response and outcome). The development of different illness phenotypes (which might not require active psychosis) may help to refine our understanding of the neurobiology of the schizophrenia spectrum and allow the development of novel treatment approaches.

\section{Relationship to age at onset}

People who develop schizophrenia therefore tend to exhibit a range of often subtle developmental abnormalities, consistent with theneurodevelopmental model of schizophrenia. It is important to note that these risk factors are non-specific (Box 4) and their use in predicting the development of schizophrenia is therefore extremely limited at present.

The presence of the risk factors detailed above appears to have some correlation with age at onset of schizophrenia. Age at onset tends to be younger in individuals at a high genetic risk for schizophrenia. Obstetric complications and impaired cognitive functioning may also be associated with earlier disease onset (Verdoux et al, 1997; Tuulio-Henriksson et al, 2004). Similarly a family history of schizophrenia, obstetric complications, low premorbid IQ and ongoing drug misuse all tend to be associated with poorer outcomes. 
Box 4 Non-specific neurodevelopmental precursors of schizophrenia

- Abnormal neuromotor development

- Delayed developmental milestones

- Soft neurological signs

- Neurocognitive deficits (verbal skills, attention, information processing)

- Neurophysiological abnormalities (eventrelated brain potentials, saccadic eye movement control)

- Poor social adjustment and school performance

\section{Implications for assessment and provision of services}

In recent years, there has been an increased emphasis on early diagnosis and intervention in psychosis. Mental health services in the UK have been reorganised, with the establishment of early intervention in psychosis teams for people aged 14-35. The establishment of these teams follows numerous studies on early intervention in psychotic illness (much of this work focusing on secondary and tertiary prevention), the aim being to improve outcomes via early recognition and treatment of symptoms (Yung et al, 1996; Birchwood et al, 1998; Larsen et al, 2001). This is underpinned by the known effects of duration of untreated psychosis (and duration of untreated illness) on outcome in schizophrenia (Perkins et al, 2005). The organisation of the services is based on the biology and pattern of onset of psychotic illness as opposed to more traditional boundaries between mental health services for adults and children.

In addition to these approaches, if the development of schizophrenia or other disorders within the schizophrenia spectrum could be predicted this would have potentially dramatic implications for assessment and treatment, including primary prevention (see Box 5).

\section{Early identification and assessment}

If risk factors for schizophrenia are known, the question arises as to whether identification of high-risk individuals is feasible and useful. As discussed above, a constellation of genetic and developmental factors are associated with later development of schizophrenia. Although the developmental findings are not specific to schizophrenia and are in themselves not sufficient grounds to offer treatment, they do raise the possibility of early assessment for highrisk individuals if symptoms occur. Examples could include the identification of people at high risk, defined according to family history of psychosis and functional impairment, or attenuated or brief psychotic symptoms, or of individuals with schizotaxia or schizotypy. Such strategies are likely to develop further in the light of further progress in our knowledge of the aetiopathology of disorders in the schizophrenia spectrum and their underlying genetic basis.

\section{Implications for the prevention of schizophrenia}

There has been interest in the possibility of employing more preventive measures for disorders within the schizophrenia spectrum for a considerable time. Although most of this work has focused on secondary prevention, there has been more recent interest in approaches based on primary prevention (Phillips et al, 2005), given the progress in our ability to identify individuals at greatest risk of developing schizophrenia. Raine et al (2003) suggested that environmental interventions in childhood could reduce measures of schizotypy later in life, which may have implications for primary prevention given the links between schizotypal personality and schizophrenia. McGorry et al (2002) performed a randomised control trial of a preventive intervention involving low-dose risperidone plus cognitivebehavioural therapy in a sample of individuals at high risk for a psychotic episode. This suggested that the intervention might be able to delay (and sometimes even prevent) the development of psychosis in very high-risk individuals. Rosen et al (2002) assessed prodromal features predictive of the development of schizophrenia and subsequently used a treatment package which included antipsychotic medication. Cornblatt et al (2003) reported an intervention which

Box 5 Issues surrounding early intervention in schizophrenia

- Identification of developmental risk factors and prodromal features raises the possibility of early intervention and prevention

- Correlation between longer duration of untreated illness and poorer outcomes

- Establishment of early intervention teams providing a range of psychological, environmental and pharmacological interventions

- Ethical issues regarding thresholds for treatment and specificity of risk factors/ symptoms

- Developments in understanding aetiopathology may lead to further advances in preventive approaches 
placed great emphasis on non-specific prodromal symptoms as well as positive symptoms, and Morrison et al (2004) suggested that cognitive therapy was an acceptable and effective intervention for reducing progression to psychosis in those at ultrahigh risk. At a population level, there has been speculation regarding the possibility of applying universal interventions for the primary prevention of schizophrenia (McGrath, 2000) by focusing on weak but prevalent risk factors.

\section{Conclusions}

The studies reported here might be seen as part of the adult literature but they have important implications for child and adolescent psychiatry, and challenge the traditional boundaries between services. The ethical implications of using these possible treatment strategies in young people will need careful consideration before their application (Phillips et $a l, 2005)$. Furthermore, the economic implications of such interventions need to be considered. Further developments in our understanding of the genetic basis and other aetiopathological factors of schizophrenia will help to improve our predictions regarding risk of schizophrenia and might make preventive interventions more feasible.

\section{Declaration of interest}

None.

\section{References}

American Psychiatric Association (1994) Diagnostic and Statistical Manual of Mental Disorders (4th edn). APA.

Andreasen, N. C. (1999) A unitary model of schizophrenia: Bleuler's "fragmented phrene" as schizencephaly. Archives of General Psychiatry, 56, 781-787.

Asarnow, J. R., Tompson, M. C. \& Goldstein, M. J. (1994) Outcome in childhood-onset schizophrenia-spectrum disorders. Schizophrenia Bulletin, 20, 599-617.

Asarnow, J. R., Tompson, M. C. \& McGrath, E. P. (2004) Childhoodonset schizophrenia: clinical issues. Journal of Child Psychology and Psychiatry, 45, 180-194.

Bellgrove, M. A, Collinson, S., Mattingley, J. B., et al (2004) Attenuation of perceptual asymmetries in patients with earlyonset schizophrenia: evidence in favour of reduced hemispheric differentiation in schizophrenia? Laterality, 9, 79-91.

Berry, N., Jobanputra, V. \& Pal, H. (2003) Molecular genetics of schizophrenia: a critical review. Journal of Psychiatry and Neuroscience, 28, 415-429.

Birchwood, M., Todd, P. \& Jackson, C. (1998) Early intervention in psychosis. The critical period hypothesis. British Journal of Psychiatry (suppl. 33), 172, 53-59.

Bleuler, E. (1911) Dementia Praecox or the Group of Schizophrenias. Reprinted 1950. International University Press.

Bracha, H. S., Torrey, E. F., Bigelow, L. B., et al (1991) Subtle signs of prenatal maldevelopment of the hand ectoderm in schizophrenia: a preliminary monozygotic twin study. Biological Psychiatry, 30, 719-725.

Cannon, T., Bearden, C. E., Hollister, J. M., et al (2000) Childhood cognitive functioning in schizophrenia patients and their unaffected siblings: a prospective cohort study. Schizophrenia Bulletin, 26, 379-393.
Cannon-Spoor, H. E., Potkin, S. G. \& Wyatt, R. J. (1982) Measurement of premorbid adjustment in chronic schizophrenia. Schizophrenia Bulletin, 8, 470-484.

Cornblatt, B. A., Lencz, T., Smith, C. W., et al (2003) The schizophrenia prodrome revisited: a neurodevelopmental perspective. Schizophrenia Bulletin, 29, 633-651.

Crow, T. J., Done, D. J. \& Sacker, A. (1995) Childhood precursors of psychosis as clues to its evolutionary origins. European Archives of Psychiatry and Clinical Neuroscience, 245, 61-69.

Davalos, D. B., Compagnon, N., Heinlein, S., et al (2004) Neuropsychological deficits in children associated with increased familial risk for schizophrenia. Schizophrenia Research, 67, 123-130.

Done, D. J., Crow, T. J., Johnstone, E. C., et al (1994) Childhood antecedents of schizophrenia and affective illnesses: social adjustment at ages 7 and $11 . B M J, 309,699-703$.

Erlenmeyer-Kimling, L., Rock, D., Roberts, S. A., et al (2000) Attention, memory, and motor skills as childhood predictors of schizophrenia-related psychoses: the New York High-Risk Project. American Journal of Psychiatry, 157, 1416-1422.

Fish, B., Marcus, J., Hans, S. L., et al (1992) Infants at risk for schizophrenia: sequelae of a genetic neurointegrative defect. A review and replicative analysis of pandysmaturation in the Jerusalem infant development study. Archives of General Psychiatry, 49, 221-235.

Frost, D. O., Tamminga, C. A., Medoff, D. R., et al (2004) Neuroplasticity and schizophrenia. Biological Psychiatry, 56, 540-543.

Gottesman, I. I. Gould, T. D. (2003) The endophenotype concept in psychiatry: etymology and strategic intentions. American Journal of Psychiatry, 160, 636-645.

Hafner, H., Maurer, K., Loffler, W., et al (1993) The influence of age and sex on the onset and early course of schizophrenia. British Journal of Psychiatry, 162, 80-86.

Harrison, P. J. \& Weinberger, D. R. (2005) Schizophrenia genes, gene expression, and neuropathology: on the matter of their convergence. Molecular Psychiatry, 10, 40-68.

Hollis, C. (2000) Adult outcomes of child- and adolescent-onset schizophrenia: diagnostic stability and predictive validity. American Journal of Psychiatry, 157, 1652-1659.

Jablensky, A., Sartorius, N., Ernberg, G., et al (1992) Schizophrenia: manifestations, incidence and course in different cultures. A World Health Organisation 10-Country Study. Psychological Medicine Monograph Supplement, 20, 1-97.

Johnstone, E. C., Ebmeier, K. P., Miller, P., et al (2005) Predicting schizophrenia: findings from the Edinburgh High-Risk Study. British Journal of Psychiatry, 186, 18-25.

Jones, P., Rodgers, B., Murray, R., et al (1994) Child development risk factors for adult schizophrenia in the British 1946 birth cohort. Lancet, 344, 1398-1402.

Kendler, K. \& Gardner, C. (1997) The risk for psychiatric disorders in relatives of schizophrenic and control probands: a comparison of three independent studies. Psychological Medicine, 27, 411-419.

Kendler, K., McGuire, M., Gruenberg, A., et al (1995) Schizotypal symptoms and signs in the Roscommon Family Study. Their factor structure and familial relationship with psychotic and affective disorders. Archives of General Psychiatry, 52, 296-303.

Keshavan, M. S., Berger, G.,Zipursky, R. B., et al (2005) Neurobiology of early psychosis. British Journal of Psychiatry, 187 (suppl. 48), s8-s18.

Kirkbride, J. B., Fearon, P., Morgan, C., et al (2006) Heterogeneity of incidence rates of schizophrenia and other psychotic syndromes. Findings from the 3-center AESOP study. Archives of General Psychiatry, 63, 250-258.

Larsen, T. K., McGlashan, T. H., Johannessen, J. O., et al (2001) Shortened duration of untreated first episode of psychosis: changes in patient characteristics at treatment. American Journal of Psychiatry, 158, 1917-1919.

Lichtermann, D., Karbe, E. \& Maier, W. (2000) The genetic epidemiology of schizophrenia and of schizophrenia spectrum disorders. European Archives of Psychiatry and Clinical Neuroscience, 250, 304-310.

Liddle, P. F. (1987) The symptoms of chronic schizophrenia: a reexamination of the positive-negative dichotomy. British Journal of Psychiatry, 151, 145-151.

McGorry, P. D., Yung, A. R., Phillips, L. J., et al (2002) Randomized control trial of interventions designed to reduce the risk of 
progression to first-episode psychosis in a clinical sample with subthreshold symptoms. Archives of General Psychiatry, 59, 921-928.

McGrath, J. (2000) Universal interventions for the primary prevention of schizophrenia. Australian and New Zealand Journal of Psychiatry, 34 (suppl.), S58-S64.

Malmberg, A., Lewis, G., David, A., et al (1998) Premorbid adjustment and personality in people with schizophrenia. British Journal of Psychiatry, 172, 308-313.

Meehl, P. E. (1989) Schizotaxia revisited. Archives of General Psychiatry, 46, 935-944.

Morrison, A. P., French, P., Walford, L., et al (2004) Cognitive therapy for the prevention of psychosis in people at ultra-high risk. British Journal of Psychiatry, 185, 291-297.

Pantelis, C., Velakoulis, D., McGorry, P. D., et al (2003) Neuroanatomical abnormalities before and after onset of psychosis: a cross-sectional and longitudinal MRI comparison. Lancet, 361 281-288.

Perkins, D. O., Gu, H., Boteva, K., et al (2005) Relationship between duration of untreated psychosis and outcome in first-episode schizophrenia: a critical review and meta-analysis. American Journal of Psychiatry, 162, 1785-1804.

Phillips, L. J., McGorry, P. D., Yung, A. R., et al (2005) Prepsychotic phase of schizophrenia and related disorders: recent progress and future opportunities. British Journal of Psychiatry, 187 (suppl. 48), s33-s44

Raine, A., Mellingen, K., Liu, J., et al (2003) Effects of environmental enrichment at ages 3-5 years on schizotypal personality and antisocial behavior at ages 17 and 23 years. American Journal of Psychiatry, 160, 1627-1635.

Rapoport, J. L., Giedd, J., Kumra, S., et al (1997) Childhood-onset schizophrenia: progressive ventricular change during adolescence. Archives of General Psychiatry, 54, 897-903.

Ridler, K., Veijola, J. M., Tanskanen, P., et al (2006) Fronto-cerebellar systems are associated with infant motor and adult executive functions in healthy adults but not in schizophrenia. Proceedings of the National Academy of Sciences USA, 103, 15651-15656.

Rosen, J. L., Woods, S. W., Miller, T. J., et al (2002) Prospective observations of emerging psychosis. Journal of Nervous and Mental Disease, 190, 133-141.

Singh, S. P., Cooper, J. E., Fisher, H. L., et al (2005) Determining the chronology and components of psychosis onset: The Nottingham Onset Schedule (NOS). Schizophrenia Research, 80, 117-130.

Tuulio-Henriksson, A., Partonen, T., Suvisaari, J., et al (2004) Age at onset and cognitive functioning in schizophrenia. British Journal of Psychiatry, 185, 215-219.

Verdoux, H., Geddes, J. R., Takei, N., et al (1997) Obstetric complications and age at onset in schizophrenia: an international collaborative meta-analysis of individual patient data. American Journal of Psychiatry, 154, 1220-1227.

Walker, E. F. (1994) Developmentally moderated expressions of the neuropathology underlying schizophrenia. Schizophrenia Bulletin, 20, 453-480.

Watt, N. F. (1978) Patterns of childhood social development in adult schizophrenics. Archives of General Psychiatry, 35, 160-165.

Weinberger, D. R., Cannon-Spoor, E., Potkin, S. G., et al (1980) Poor premorbid adjustment and CT scan abnormalities in chronic schizophrenia. American Journal of Psychiatry, 137, 1410-1413.

Yung, A. R., McGorry, P. D., McFarlane, C. A., et al (1996) Monitoring and care of young people at incipient risk of psychosis. Schizophrenia Bulletin, 22, 283-303.

\section{MCQs}

1 Individuals with schizotypy:

a often show poor social interactions

b do not experience psychotic symptoms

c will eventually develop schizophrenia

d do not share common genetic vulnerabilities with schizophrenia

e are not considered as having a schizophrenia-spectrum disorder.
2 Genetic studies:

a suggest that schizophrenia has a small genetic component

b provide evidence inconsistent with the neurodevelopmental hypothesis of schizophrenia

c do not support the concept of schizotaxia

d suggest that genetic predisposition always leads to schizotypy or schizophrenia

e have implicated genes involved in synaptic function in determining susceptibility to schizophrenia.

3 In childhood:

a a diagnosis of schizophrenia does not have predictive validity

b outcome of childhood-onset schizophrenia is better than that of adult-onset schizophrenia

c schizotypal features cannot occur

d younger age correlates with higher thought disorder scores

e interventions do not have any effect on measures of schizotypy in adult life.

4 Developmental factors associated with schizophrenia:

a always result in the later development of the disorder

b are specific

c may involve late neurodevelopmental influences as well as early influences

d do not include abnormal motor development

e do not include poor social adjustment and educational difficulties.

5 Identification of developmental factors associated with schizophrenia and early interventions:

a will not affect our ability to predict those at risk of developing schizophrenia

b has no implications for preventive treatments

c may place an emphasis on interventions for non-specific prodromal symptoms

$\mathrm{d}$ has indicated that cognitive-behavioural therapy is not an effective treatment strategy

e has indicated that early interventions have no effect on clinical outcomes. 